\title{
FAMILIES OF MEASURES AND REPRESENTATIONS OF ALGEBRAS OF OPERATORS
}

\author{
BY \\ E. J. McSHANE
}

Introduction. The purpose of the present paper is most easily explained by examining one part of it in detail. Let $\left\{B_{\omega}: \omega \in \Omega\right\}$ be a set of commuting bounded hermitian operators on a Hilbert space $H$, and let $T$ be the cartesian product $X_{\omega \in \Omega}\left[-\left\|B_{\omega}\right\|,\left\|B_{\omega}\right\|\right]$. Two of the most useful tools in the study of the operators are the simultaneous spectral resolution of the $B_{\omega}$ by means of a projector-valued measure $\pi$ on a $\sigma$-field $a$ of subsets of $T$, and the multiplicative representation. For this last we choose a set $\left\{x_{j}: j \in J\right\}$ of vectors in $H$ such that if $H\left(x_{j}\right)$ is the closed linear extension of the set $\left\{\pi(A) x_{j}: A \in Q\right\}$, the $H\left(x_{j}\right)$ are mutually orthogonal and their closed linear extension is $H$. Then $H$ is the direct sum of the $H\left(x_{j}\right)$, and for each $j$ in $J$ there is a nonnegative measure $m_{j}$ on $a$ such that $L_{2}\left[T, m_{j}\right]$ is isomorphic with $H\left(x_{j}\right)$, and for every (complex) bounded Baire function $f$ on $T$ the operator $f\left(B_{w}\right)$ $=\int f(t) d \pi$ corresponds in the direct sum $H_{0}=\sum_{j} \oplus L_{2}\left[T, m_{j}\right]$ to the multiplication operator that maps $(\phi(t, j): t \in T, j \in J)$ on $(f(t) \phi(t, j): t \in T, j \in J)$. If $B$ is the smallest weakly (or strongly) closed commutative self-adjoint algebra of operators that contains all the $B_{\omega}$, the operators thus defined by multiplication form a subalgebra of $B$; but this may be a proper subalgebra and may fail to be weakly closed. On the other hand, if for every bounded complex function $(f(t, j): t \in T, j \in J)$ which is a-measurable on $T$ for each $j$ in $J$ we construct the operator that maps the vector $(\phi(t, j): t \in T, j \in J)$ of the direct sum $H_{0}$ on

$$
(f(t, j) \phi(t, j): t \in T, j \in J) .
$$

we obtain too many operators; there are such operators which, under the isomorphism of $H_{0}$ and $H$, do not correspond to members of $B$.

One of our principal results is that if we suitably restrict the functions $f$ of the preceding sentence we obtain the multiplicative representation of the algebra $B$ itself. The functions $f$ allowed are those which depend on $j$ only in the following heavily restricted way. To each countable subset $J_{c}$ of $J$ there corresponds an $Q$-measurable function $(f(t): t \in T)$ such that for each $j$ in $J_{c}, f(t)=f(t, j)$ except on a set whose $m_{j}$-measure is 0 . Such functions on $T \times J$ we call "quasi-functions on $T$." For such functions $f$ we can also define $\int_{T} f d \pi$, by first projecting each vector $x$ of $H$ into the subspaces $H\left(x_{j}\right)$, applying $\int f(t, j) d \pi$ to the projection on $H\left(x_{j}\right)$, and adding the results. The integrals thus formed constitute an algebra of operators, and this algebra is

Presented to the Society, March 13, 1961; received by the editors March 13, 1961. 
exactly the smallest weakly closed self-adjoint algebra of operators containing all the $B_{\omega}$. It is worth noticing that if $J$ is countable we can replace $f(t, j)$ by the $f(t)$ of the definition (with $J_{c}=J$ ); the "new" integral is then the standard $\int f(t) d \pi$, and we conclude that if the cyclic set $\left\{x_{j}: j \in J\right\}$ is countable the algebra of operators $f\left(B_{w}\right)$ defined by the integral is weakly closed, even though $\Omega$ may be uncountable and $H$ nonseparable.

The second part of this paper is devoted to showing that the "quasifunctions on $T^{\prime \prime}$ have this desirable property, and that they also have somewhat similar uses in extending the Radon-Nikodym theorem and the Riesz representation theorem. [Since this was written, A. C. Zaanen has presented to the American Mathematical Society a paper in which the Radon-Nikodym theorem is extended just as it is here. The "quasi-functions" appropriate to this situation are called "cross-sections" by Zaanen. See abstract, Notices Amer. Math. Soc. 7, no. 7 (1960), 986; his paper will appear( $\left.{ }^{1}\right)$ in the Nederl. Akad. Wetensch. Proc. Ser. A.] The first part of the paper consists largely of the extension (to infinite sets of measure-functions) of a few of the results obtained by I. E. Segal [2] for (single) "localizable" measures. My apology for presenting these extensions is that they are needed in the second part.

1. Measures. Here and henceforth $T$ will denote a set and $Q$ a $\sigma$-ring of subsets of $T . J$ will denote a nonempty set, used only for indices to distinguish objects. For each $j$ in $J, m_{j}$ is a non-negative extended-real-valued function on $a$, and is countably additive. If $F \subset T$ and $j \in J, F$ is said to be $m_{j}$-finite if $F \in Q$ and $m_{j} F<\infty$, and $F$ is said to be $m_{j}$-null if $F \in Q$ and $m_{j} F=0$. For brevity, we use the expression " $F$ is $m_{J}$-finite" to mean that $F$ is $m_{j}$-finite for all $j$ in $J$, while " $F$ is $m_{J}$-null" means that $F$ is $m_{j}$-null for all $j$ in $J$. The class of all $m_{J}$-finite sets will be denoted by $R$.

We use a device of I. E. Segal's [2] to eliminate the nuisance of sets of infinite measure that contain no subset of finite positive measure. We define $A^{\prime}$ to be the collection of all subsets $E$ of $T$ such that $E \cap F \in R$ for all $F$ in $\Re$. This is a $\sigma$-ring, and is in fact a $\sigma$-algebra since $T$ is in it. Next, for each $j$ in $J$ and each $E$ in $Q^{\prime}$ we define $m_{j}^{\prime} E$ to be the supremum of $m_{j}(E \cap F)$ for all $F$ in $R$. These new measures are countably additive on $Q^{\prime}$, and $m_{j}^{\prime} F=m_{j} F$ for all $j$ in $J$ and all $F$ in $R$. If we treat $a^{\prime}$ and $\left\{m_{j}^{\prime}: j \in J\right\}$ as we treated $a$ and $\left\{m_{j}: j \in J\right\}$ we obtain them back again. It is with $Q^{\prime}$ and the measures $m_{j}^{\prime}$ that we work henceforth. We drop the prime from the notation, and we list the properties henceforth assumed.

(1.1) $a$ is an algebra of subsets of a set $T$.

For each $j$ in a set $J, m_{j}$ is a non-negative extended-real-valued countably additive measure in $a$.

$R$ is the set of all $m_{J}$-finite sets (i.e., all $F$ in a with $m_{j} F<\infty$ for all $j$ in $J$ ).

For all subsets $S$ of $T, S \in Q$ if and only if $S \cap F \in R$ for all $F$ in $R$, and in that case $m_{j} S=\sup \left\{m_{j}(S \cap F): F \in R\right\}$ for every $j$ in $J$.

(1) See reference [6]. 
A set $S$ is measurable if it belongs to $a$. An extended-real-valued function $f$ on $T$ is measurable if $f^{-1}(I) \in Q$ for every interval $I$ in $[-\infty, \infty]$.

To aid in remembering the domains of functions, we denote the identity function on $T$ by $t$ and the identity function on $J$ by $j$. Functions will be assumed to be extended-real-valued unless the contrary is stated. Thus $g(t, j)$ will denote a function $g: T \times J \rightarrow[-\infty, \infty]$.

2. Localizability. A measure $m_{j}$ will be called localizable (by a small modification of the definition in [2]) if

(2.1) to each nonempty collection $\nVdash$ of measurable sets there corresponds a supremum $B$ in the sense that

(i) for each $S$ in $K, S-B$ is $m_{j}$-null;

(ii) if $B^{\prime} \in Q$ and $S-B^{\prime}$ is $m_{j}$-null for every $S$ in $\mathfrak{K}$, so is $B-B^{\prime}$.

We shall say that

(2.2) $a$ set $B$ is a simultaneous supremum of the collection $K$ of sets with respect to the measures $m_{j}(j \in J)$, or more briefly that $B$ is an $m_{J}$-supremum of $\mathcal{K}$, if

(i) for each $S$ in $\mathcal{K}$ and each $j$ in $J, S-B$ is $m_{j}$-null;

(ii) for each $j$ in $J$ and each $B^{\prime}$ in $a$, if $m_{j}\left(S-B^{\prime}\right)=0$ for every $S$ in $\Re$ then $m_{j}\left(B-B^{\prime}\right)=0$.

Likewise,

(2.3) if $\mathcal{F}$ is a collection of measurable functions on $T$, a function $b(t)$ is a simultaneous supremum of $\mathcal{F}$ with respect to the measures $m_{j}(j \in J)$, or more briefly an $m_{\mathrm{J}}$-supremum of $\mathcal{F}$, if

(i) for each $f$ in $\mathcal{F}$ and each $j$ in $J, b(t) \geqq f(t)$ except on an $m_{j}-n u l l$ set;

(ii) if $b^{\prime}(t)$ is measurable and $j$ is in $J$, and for each $f$ in $F$ it is true that $b^{\prime}(t) \geqq f(t)$ except on an $m_{j}$-null set, then $b^{\prime}(t) \geqq b(t)$ except on an $m_{j}$-null set.

The measures $m_{j}(j \in J)$ are simultaneously localizable if every nonempty collection $\mathcal{K}$ of measurable sets has a simultaneous supremum with respect to the measures $m_{j}(j \in J)$. This immediately implies an analogous property for functions, as follows.

(2.4) Corollary. Let the measures $m_{j}(j \in J)$ be simultaneously localizable. Let $\mathcal{F}$ be a nonempty collection of measurable functions. Then $\mathcal{F}$ has an $m_{J}$ supremum, and it is measurable.

For each rational number $r$ let $\mathcal{K}_{r}$ consist of the measurable sets $\{t: t \in T, f(t) \geqq r\}$ with $f$ in $\mathcal{F}$, and let $B_{r}$ be the $m_{\mathrm{J}}$-supremum of the class $\aleph_{r}$. If $r^{\prime}>r, B_{r^{\prime}}-B_{r}$ is $m_{J}$-null, so the set $S_{r}=U\left\{B_{r^{\prime}}: r^{\prime}\right.$ rational, $\left.r^{\prime} \geqq r\right\}$ differs from $B_{r}$ by an $m_{J}$-null set. If $r>r^{\prime}, S_{r^{\prime}} \supset S_{r}$. Define $b_{r}(t)$ by setting $b_{r}(t)=r$ if $t \in S_{r}, b_{r}(t)=-\infty$ otherwise; and define $b(t)=\sup \left\{b_{r}(t): r\right.$ rational $\}$. This is measurable. Let $f$ belong to $F$, and for rational $r$ define $E_{r}=\{t: f(t)>r>b(t)\}$. Then $E_{r} \subset\{t: t \in T, f(t) \geqq r\} \in \mathcal{K}_{r}$, so $E_{r}-B_{r}$ is $m_{J}$-null, and therefore so is $E_{r}-S_{r}$. But $E_{r} \cap S_{r}$ is empty by definition of $b(t)$, so $E_{r}$ is $m_{J}$-null. The set on which $f(t)>b(t)$ is contained in the $m_{J}$-null set $U\left\{E_{r}: r\right.$ rational $\}$, and (2.3i) holds. 
Now let $j$ be in $J$ and let $b^{\prime}$ be as in (2.3ii), and for rational $r$ define $B_{r}^{\prime}=\left\{t: t \in T, b^{\prime}(t) \geqq r\right\}$. For each $f$ in $F, B_{r}^{\prime}$ contains all of $\{t: t \in T, f(t) \geqq r\}$ except an $m_{j}$-null subset. By $(2.2 \mathrm{ii}), m_{j}\left(B_{r}-B_{r}^{\prime}\right)=0$, so $m_{j}\left(S_{r}-B_{r}^{\prime}\right)=0$. Hence $N=\cup\left\{S_{r}-B_{r}^{\prime}: r\right.$ rational $\}$ is $m_{j}$-null. If $t \in T-N$,

$$
\begin{aligned}
b(t) & =\sup \left\{r: r \text { rational, } t \in S_{r}\right\} \\
& \leqq \sup \left\{r: r \text { rational, } t \in B_{r}^{\prime}\right\} \\
& =b^{\prime}(t),
\end{aligned}
$$

and (2.3ii) holds.

3. Simultaneous localizability. We now seek conditions that will ensure that the measures in a set $\left\{m_{j}: j \in J\right\}$ are simultaneously localizable.

(3.1) Theorem. If $J$ is countable and $T$ is $m_{J}$-finite, the measures $m_{j}(j \in J)$ are simultaneously localizable.

Let $\mathcal{K}$ be a nonempty collection of measurable sets, $\mathcal{K}^{\prime}$ the collection of all finite unions of sets in $\Re$. We may suppose that $J$ is a subset of the positive integers. For each $j$ in $J$ let $b_{j}=\sup \left\{m_{j} K: K \in \mathcal{K}^{\prime}\right\}$. For each positive integer $n$ there is a $K_{n}$ in $K^{\prime}$ such that

$$
b_{j}-1 / n \leqq m_{j} K_{n} \leqq b_{j} \quad(j \in J, j \leqq n) ;
$$

and we may suppose that $K_{n}$ increases with $n$. Define $B=U_{n} K_{n}$. If $K \in \mathscr{K}$, $K_{n} \cup K \in K^{\prime}$ for all $n$, so for each $j$ in $J m_{j}\left(K_{n} \cup K\right) \leqq b_{j}$, whence $m_{j}(B \cup K)$ $\leqq b_{j}=m_{j} B$. Thus $m_{j}(K-B)=0$, and (2.2i) holds. If $j \in J$ and $B^{\prime}$ is measurable and $m_{j}\left(K-B^{\prime}\right)=0$ for all $K$ in $\mathcal{K}$, then $m_{j}\left(K_{n}-B^{\prime}\right)=0$ for all $n$, and $m_{j}\left(B-B^{\prime}\right)$ $=0$, so (2.2ii) holds.

If $m$ is a measure on the $\sigma$-ring $a$, and $E \in a$, we define the "restriction of $m$ to $E$ " to be the measure defined by

(3.2) $m_{E} S=m(E \cap S)$ for all $S$ in $a$.

If $m$ has the properties (1.1) so has $m_{E}$.

(3.3) Theorem. Let (1.1) hold. Assume that there is a disjoint collection 8 of measurable subsets of $T$ whose union is $T$ and which satisfy

(i) for each $E$ in $\varepsilon$, the restrictions $m_{j, E}(j \in J)$ are simultaneously localizable;

(ii) for each subset $S$ of $T, S \in Q$ if and only if $S \cap E \in Q$ for every $E$ in $\varepsilon$;

(iii) if $j \in J$ and $S \in Q$ and $m_{j} S>0$, there exists an $E$ in $\&$ for which $m_{j}(E \cap S)>0$.

Then the measures $m_{j}(j \in J)$ are simultaneously localizable.

Let $\mathfrak{K}$ be a nonempty collection of measurable sets. For each $E$ in $\varepsilon$, let $B_{E}$ be the $m_{J, E}$-supremum of $\mathcal{K}$; we may assume $B_{E} \subset E$. Let $B$ be the union of the $B_{E}$; by (ii) this is measurable. If $K \in \mathcal{K}, m_{j, E}(K-B)=0$ for all $j$ in $J$ and all $E$ in $\mathcal{E}$, so by (iii) $K-B$ is $m_{\mathrm{s}}$-null. Thus (2.2i) holds, and (2.2ii) is proved in practically the same way.

(3.4) Remark. The hypothesis in Theorem 3.3 that $\mathcal{E}$ is a disjoint collec- 
tion can be weakened to:

Each set $E$ in $\&$ has points in common with at most countably many other sets in $\varepsilon$.

We well-order $\varepsilon$ by a relation $\prec$, and for each $E$ in $\varepsilon$ we define $E^{\prime}=E$ $-\cup\left\{E^{\prime \prime}: E^{\prime \prime} \in \mathcal{E}, E^{\prime \prime} \prec E\right\}$. These sets $E^{\prime}$ are measurable and disjoint, and each $E_{0}$ in $\varepsilon$ is the union of countably many sets $E_{0} \cap E^{\prime}$, so the collection of $E^{\prime}$ satisfies the requirements of Theorem 3.3.

(3.5) CoROllary. If $T$ is the union of a countable collection $\&$ of measurable sets, and for each $L$ in $\mathscr{L}$ the restrictions $m_{j, L}(j \in J)$ are simultaneously localizable, then the $m_{j}(j \in J)$ are simultaneously localizable.

As a special case, if $J$ is countable and $T$ is the union of countably many $m_{J}$-finite sets, the $m_{j}$ are simultaneously localizable. Still more particularly, every $\sigma$-finite measure is localizable.

(3.6) Corollary. If $J$ is countable, and there exists a collection $\&$ of $m_{J^{-}}$ finite sets whose union is $T$ and no one of which has points in common with more than countably many other members of $\mathcal{E}$, and for each $j$ in $J$ and each $S$ in $a$ such that $m_{j} S>0$ there is an $E$ in $\&$ such that $m_{j}(S \cap E)>0$, then the measures $m_{j}(j \in J)$ are simultaneously localizable.

By the proof of (3.4) we may assume that $\varepsilon$ is disjoint, and by Theorem 3.1, (3.3i) holds. For each $m_{J}$-finite set $F$ let $\varepsilon[F]$ be the (countable) set of $E$ in $\varepsilon$ such that $m_{j}(E \cap F)>0$ for at least one $j$ in $J$, and let $D_{F}$ be the (measurable) set $F-\cup\{F \cap E: E \in \mathcal{E}[F]\}$. Clearly $D_{F} \cap E$ is $m_{J}$-null for all $E$ in $\varepsilon$, so $D_{F}$ is $m_{J}$-null. If $S \subset T$ and $S \cap E \in Q$ for all $E$ in $\varepsilon$, then whenever $F$ is $m_{J}$-finite $S \cap F$ is the union of the countably many measurable sets $S \cap E \cap F(E \in \mathcal{E}[F])$ and the $m_{J}$-null set $S \cap D_{F}$, so by (1.1) $S \in Q$. In particular, $T-\cup \mathcal{E}$ is $m_{J}$-null, and we may adjoin it to any one set of $\varepsilon$ without disturbing the requirements. Now by Theorem 3.3 the $m_{j}(j \in J)$ are simultaneously localizable.

4. A decomposition theorem. The results of $\$ 3$ suggest the following question. If a measure $m$ is localizable, can $T$ be subdivided into a disjoint collection of sets of finite measure in such a way that (3.3ii, iii) are satisfied? We partially answer this in Theorem 4.2. We consider countably many measures $m_{j}$ simultaneously, since this creates no added difficulty.

(4.1) Lemma. Let $J$ be countable. Let $\varepsilon^{*}$ be a maximal family of $m_{J}$-finite subsets of $T$, such that no set in $\mathcal{E}^{*}$ is $m_{\mathrm{J}}$-null and each pair of distinct members of $\varepsilon^{*}$ has $m_{J}$-null intersection. Then

(i) $T$-UE* is $m_{J}-n u l l$;

(ii) if $S \subset T, S \in a$ if and only if $S \cap E \in$ a for every $E$ in $\mathcal{E}^{*}$;

(iii) for each $j$ in $J$ and each $S$ in a such that $m_{j} S>0$, there exists an $E$ in $\mathcal{E}^{*}$ such that $m_{j}(S \cap E)>0$. 
For each $m_{J}$-finite set $F$ define $\varepsilon^{*}[F]$ to be the (countable) set of $E$ in $\mathcal{E}^{*}$ with $F \cap E$ not $m_{J}$-null; then $D_{F}=F-\cup\left\{E \cap F: E \in \mathcal{E}^{*}[F]\right\}$ is measurable. Then for $E$ in $\varepsilon^{*}, D_{F} \cap E$ is $m_{J}$-null; and by the maximality of $\varepsilon^{*}, D_{F}$ must be $m_{J}$-null. Now let $S$ be a subset of $T$ such that $S \cap E \in Q$ for all $E$ in $\varepsilon^{*}$. For every $m_{J}$-finite set $F, S \cap F$ is the union of countably many measurable sets $S \cap F \cap E\left(E \in \mathcal{E}^{*}[F]\right)$ and the $m_{J}$-null set $S \cap D_{F}$, so $S \cap F \in Q$. By (1.1), $S \in$ Q. In particular, if $j \in J$ and $m_{j} S>0$, there is an $m_{J}$-finite set $F$ for which $m_{j}(S \cap F)>0$, and one of the countably many sets $S \cap F \cap E\left(E \in \mathcal{E}^{*}[F]\right)$ must have positive $m_{j}$-measure; then $m_{j}(S \cap E)>0$. This implies that $T-U \varepsilon^{*}$ is $m_{\mathrm{J}}$-null, completing the proof.

The Hausdorff maximality principle allows us to prove easily that sets $\mathcal{E}^{*}$ satisfying the hypotheses of (4.1) exist. Segal uses this [2, p. 287] to show that there exists a measure-preserving map of the measurable sets in $T$ onto measurable sets in another measure-space under which images of sets in $\mathcal{E}^{*}$ are disjoint. However, this does not provide a decomposition of $T$ itself. If $\mathcal{E}^{*}$ is countable it is trivial to construct another family whose members are disjoint. We now show that such a construction is possible whenever there are at most continuum-many sets in $\mathcal{E}^{*}$.

(4.2) Theorem. Let the measures $m_{j}(j \in J)$ be simultaneously localizable. If $J$ is countable, and there exists a family $\varepsilon^{*}$ satisfying the hypotheses of Lemma 4.1 and having cardinal number not greater than that of the continuum, there exists a decomposition $\&$ of $T$ with the properties (ii) and (iii) of (3.3), the sets in $\&$ being $m_{J}$-finite and mutually disjoint.

There is a mapping $r$ of $\varepsilon^{*}$ onto a subset $R_{0}$ of the open interval $(0,1)$. Let $\mathcal{F}$ be the set of functions $f_{E *}\left(E^{*} \in \mathcal{E}^{*}\right)$, where $f_{E^{*}}$ has value $r\left(E^{*}\right)$ on $E^{*}$ and value 0 on $T-E^{*}$. By Corollary $2.4, \mathcal{F}$ has a supremum $b(t)$. Then $b(t) \geqq 0$ except on an $m_{J}$-null set, and we may suppose it non-negative on $T$. Let $E^{\prime}$ be in $\varepsilon^{*}$; define $h(t)$ by

$$
h(t)=r\left(E^{\prime}\right)\left(t \in E^{\prime}\right) ; \quad h(t)=b(t)\left(t \in T-E^{\prime}\right) .
$$

For each $E^{*}$ in $\varepsilon^{*}$ let $D_{E^{*}}=\left\{t: t \in T, f_{E^{*}}(t)>h(t)\right\}$. If $E^{*}=E^{\prime}$ this is empty; if $E^{*} \neq E^{\prime}, D_{E^{*}} \cap\left[T-E^{\prime}\right]$ is contained in the $m_{J}$-null set $\left\{t: t \in T, f_{E^{*}}(t)>b(t)\right\}$ and $D_{E^{*}} \cap E^{\prime}$ is contained in the $m_{J}$-null set $E^{*} \cap E^{\prime}$, so in all cases $D_{E^{*}}$ is $m_{J}$-null. By definition of $m_{J}$-supremum, $h(t) \geqq b(t)$ except on an $m_{J}$-null set. In particular, the subset of $E^{\prime}$ on which $h(t) \geqq b(t)$ fails to hold is $m_{J}$-null. The reversed inequality is obvious, so $b(t)=r\left(E^{\prime}\right)$ on all of $E^{\prime}$ except an $m_{J^{-}}$ null subset.

Now for each $r$ in $R_{0}$ we define $E(r)=b^{-1}(r)$, and we define $\varepsilon$ to be $\left\{E(r): r \in R_{0}\right\}$. The mapping $E^{*} \rightarrow E\left(r\left(E^{*}\right)\right)$ is a one-to-one correspondence between $\varepsilon^{*}$ and $\varepsilon$, and corresponding sets differ by $m_{J}$-null sets. Thus $\varepsilon$ is a disjoint collection of sets none of which is $m_{J}$-null. The set $\varepsilon$ satisfies the hypotheses placed upon $\varepsilon^{*}$ in Lemma 4.1 . Thus $T-U \varepsilon$ is $m_{J}$-null; we adjoin 
it to any one set of $\varepsilon$, and the amended $\varepsilon$ has union $T$. The other conclusions hold by Lemma 4.1 .

5. Definition and lattice properties. We continue to assume that (1.1) is satisfied.

(5.1) A function $f(t, j)$ will be called a "quasi-function on $T$ " with respect to the measures $m_{j}(j \in J)$ if to each countable subset $J_{c}$ of $J$ there corresponds a function $f(t)$ such that $m_{j}\{t: t \in T, f(t, j) \neq f(t)\}=0$ for every $j$ in $J_{c}$.

Occasionally we shall use the symbol $f(t, *)$ for a quasi-function; the use of the asterisk in place of $j$ shall connote the relation between the various $f(t, j)$ specified in the definition of quasi-function. A quasi-function $f(t, *)$ is called measurable if all the functions $f(t, j)(j \in J)$ are measurable.

Each function $f(t)$ defines a quasi-function $f(t, *)$ such that $f(t, j)=f(t)$ for all $t$ in $T$ and all $j$ in $J$. Conversely, if $f(t, j)$ is independent of $j$ it defines a function on $T$. By a familiar abuse of language we then say that the quasifunction $f(t, *)$ is the function on $T$ that it defines.

Algebraic and lattice operations on quasi-functions need no discussion, since each quasi-function is an extended-real-valued function on $T \times J$. Two quasi-functions $f(t, *), g(t, *)$ are equivalent if for each $j$ in $J$ we have $m_{j}\{t: t \in T, f(t, j) \neq g(t, j)\}=0$. Clearly, if $J$ is countable every quasi-function is equivalent to a function on $T$.

Let $\mathcal{F}$ be a nonempty set of quasi-functions on $T$, with respect to the $m_{j}(j \in J)$. Then:

(5.2) A quasi-function $b(t, *)$ is called an $m_{J}$-supremum of $\mathcal{F}$ if

(i) for each $j$ in $J$ and each $f$ in $F, b(t, j) \geqq f(t, j)$ except on an $m_{j}$-null set;

(ii) for each $j$ in $J$, if $b^{\prime}(t)$ is a function on $T$ such that for every $f$ in $\mathcal{F}$ the inequality $b^{\prime}(t) \geqq f(t, j)$ holds except on an $m_{j}$-null set, then $b^{\prime}(t) \geqq b(t, j)$ except on an $m_{j}$-null set.

Evidently any two $m_{J}$-suprema of a set $\mathcal{F}$ are equivalent.

Quasi-functions have lattice properties stronger than those of ordinary functions, as the following theorem shows.

(5.3) Theorem. Let (1.1) hold, and for each countable subset $J_{c}$ of $J$ let the measures $m_{j}\left(j \in J_{c}\right)$ be simultaneously localizable. Then each nonempty collection $\mathcal{F}$ of measurable quasi-functions on $T$ (with respect to the measures $m_{j}$, $j \in J)$ has an $m_{J}$-supremum, and this is a measurable quasi-function.

By Corollary 2.4 , for each $j$ in $J$ there is a function $b(t, j)$ satisfying $(5.2 \mathrm{i}, \mathrm{ii})$. It remains to show that $b(t, j)$ is a quasi-function. Let $J_{c}$ be a countable subset of $J$. For each $f(t, j)$ in $\mathcal{T}$ we can and do choose an $f(t)$ such that for all $j$ in $J_{c}, f(t)=f(t, j)$ except on an $m_{j}$-null set. Denote by $\mathcal{F}_{c}$ the set of $f(t)$ thus chosen. By Corollary $2.4, F_{c}$ has a supremum $b(t)$ with respect to the measures $m_{j}\left(j \in J_{c}\right)$. For each $j$ in $J_{c}$ we have $b(t, j) \geqq f(t, j)$ except on an $m_{j}$-null set whenever $f(t, j) \in \mathcal{F}$, so $b(t, j) \geqq f(t)$ except on an $m_{j}$-null set whenever $f(t) \in F_{c}$, and so $b(t, j) \geqq b(t)$ except on an $m_{j}$-null set. On the other hand, 
if $f(t, j) \in \mathcal{F}$, we have $b(t) \geqq f(t)=f(t, j)$ except on an $m_{j}$-null set, whence by (5.2ii) $b(t) \geqq b(t, j)$ except on an $m_{j}$-null set. Thus for all $j$ in $J_{c}$ we have $b(t, j)=b(t)$ except on an $m_{j}$-null set, and the proof is complete.

6. Summable quasi-functions. Suppose that we have a system satisfying (1.1) with $J$ a singleton; we then drop the subscript $j$ from $m_{j}$. Let $Q$ be the collection of $m$-finite sets, and $\mathscr{L}$ the collection of all measurable subsets $L$ of $T$ such that the restriction of $m$ to $L$ is localizable (cf. (3.2)). Then the system $\left\{T, a,\left\{m_{R}: R \in \mathbb{R}\right\}\right\}$ satisfies (1.1), and so does $\left\{T, a,\left\{m_{L}: L \in \mathcal{L}\right\}\right\}$. If we define $L$ and $R$ to be the identity functions on $\mathscr{L}$ and $R$ respectively, we can construct two classes of quasi-functions, $f(t, L)$ and $f(t, R)$. Since $\mathfrak{R} \subset \mathscr{L}$, if $f(t, L)$ is a quasi-function with respect to the measures $m_{L}(L \in \mathscr{L})$, its restriction $f(t, R)$ to $T \times R$ is a quasi-function with respect to the measures $m_{R}(R \in Q)$. Conversely, every quasi-function with respect to the $m_{R}$ can be extended to a quasi-function with respect to the $m_{L}$, as we now show.

(6.1) LеммA. If $f(t, R)$ is a quasi-function with respect to the measures $m_{R}(R \in R)$, it has an extension $f(t, L)$ to $T \times \mathfrak{L}$ which is a quasi-function with respect to the measures $m_{L}(L \in \mathscr{L})$. In particular, if $m$ is localizable $f(t, R)$ is equivalent to a measurable function $f(t)$ on $T$.

For each $R$ in $R$ let $g_{R}$ be the function coinciding with $f\left(t, R_{R}\right)$ on $R$ and identically $-\infty$ on $T-R$. By Corollary 3.5 the hypotheses of Theorem 5.3 are satisfied with $J=\mathscr{L}$, so the set $\left\{g_{R}: R \in \Omega\right\}$ has a supremum $b(t, L)$ among the measurable quasi-functions with respect to the $m_{L}(L \in \mathcal{L})$. Let $R_{1}$ and $R_{2}$ be in $R$. Then there is a measurable function $f(t)$ such that $f(t)=f\left(t, R_{i}\right)$ except on an $m_{R_{i}}$-null set $(i=1,2)$. Hence $g_{R_{1}}(t)=g_{R_{2}}(t)$ at all points of $R_{1} \cap R_{2}$ except those of an $m$-null subset. This implies $g_{R_{1}}(t) \geqq g_{R_{2}}(t)$ at all points of $T$ except an $m_{R_{1}}$-null set, and since $R_{2}$ is any member of $R, g_{R_{1}}(t) \geqq b\left(t, R_{1}\right)$ except on an $m_{R_{1}}$-null set. The reversed inequality is an immediate consequence of the definition of $b$, so $f\left(t, R_{1}\right)=g_{R_{1}}(t)=b\left(t, R_{1}\right)$ except on an $m_{R_{1}}$-null set. Without loss of generality we may assume that $b\left(t, R_{1}\right)$ was already defined so as to be identical with $f\left(t, R_{1}\right)$ for all $R_{1}$ in $R$. Then $b(t, L)$ is an extension of $f(t, R)$ to $T \times L$, and is a quasi-function with respect to the measures $m_{L}(L \in \mathscr{L})$. In particular, if $m$ is localizable, $T$ is in $\mathscr{L}$ and $m=m_{T}$; and $b(t, T)$ is a function on $T$ equivalent to $b(t, L)$. This completes the proof.

7. An extension of the Radon-Nikodym theorem. Let $f(t, R)$ be a quasifunction with respect to the $m_{R}(R \in R)$; and suppose that for each $R$ in $R$, $f(t, R)$ is $m_{R}$-summable over $T$ (or, equivalently, $m$-summable over $R$ ). Then $\int f\left(t, R^{\prime}\right) d m_{R}(t)$ has the same value for all $R^{\prime}$ in $R$ that contain $R$. This integral is said to have a limit $k$ (in $[-\infty, \infty]$ ) as $R$ expands if to each neighborhood $U$ of $k$ there corresponds an $R_{U}$ in $R$ such that whenever $R \in R$ and $R \supset R_{U}$, $\int f(t, R) d m_{R}(t) \in U$. In this case we write

$$
\int f(t, *) d m(t)=k
$$


(All integrals are over $T$.) If $f$ is non-negative the limit will surely exist; and in fact we can relax the requirement that $f(t, R)$ be $m_{R}$-summable and require that it have an integral, finite or infinite. It is easy to see that on the class of summable quasi-functions (those having finite integrals) the integral is linear, and the class is a vector lattice.

Now consider a system satisfying (1.1) with $J=\{1,2\}$. To save subscripts we write $m$ for $m_{1}$ and $n$ for $m_{2}$. (The assumption that $n$ is non-negative is not vital, but saves some bother.) $\&$ will be the class of sets $L$ in $Q$ such that $m_{L}$ is localizable.

(7.1) Theorem. Let $m$ and $n$ be as just described. A ssume that for each $R$ in $R$, if $m R=0$ then $n R=0$. Then there exists a non-negative finite valued measurable quasi-function $f(t, L)$ such that for all $S$ in $a$,

$$
n S=\int f(t, *) K_{S}(t) d m(t) .
$$

If $m$ is localizable $f(t, *)$ can be taken to be a measurable function $f(t)$ on $T$.

If $S$ is $\sigma$-finite, by the standard form of the Radon-Nikodym theorem ([1], p. 128) there is a measurable real-valued function $f$ on $S$ such that for every measurable subset $E$ of $S$,

$$
n(E)=\int_{E} f(t) d m(t) .
$$

For each $\sigma$-finite set $S$ in $a$ we choose such an integrand, and we define $f_{S}(t)$ to be $f(t)$ for $t$ in $S$ and to be 0 for $t$ in $T-S$. These $f_{S}$ may be regarded as quasi-functions on $T$ with respect to the $m_{L}(L \in \mathcal{L})$, and as such they have an $m_{\mathscr{L}}$-supremum $f(t, L)$, which we may suppose to be everywhere nonnegative.

If $R$ is in $R$ it is in $\mathscr{L}$, so the inequality $f(t, R) \geqq f_{R}(t)$ holds except on an $m_{R}$-null set. If $S$ is $\sigma$-finite, $f_{S}$ and $f_{R}$ have the same integral $n(E)$ over every measurable subset $E$ of $R \cap S$, so they coincide on all of $R \cap S$ except an $m$ null set. But $f_{S}$ vanishes on $R-S$, so $f_{R} \geqq f_{S}$ except on an $m_{R}$-null set. By definition of supremum, $f_{R} \geqq f(t, R)$ except on an $m_{R}$-null set. With the previous inequality, this proves that $f_{R}(t)=f(t, R)$ except on an $m_{R}$-null set. So for all $S$ in $Q$

$$
n(R \cap S)=\int f(t, R) K_{S}(l) d m_{R}(t)
$$

The integral increases as $R$ expands, so for fixed $S$ in $Q$ its limit as $R$ expands is the same as its supremum for all $R$ in $R$, whence

$$
n S=\int f(t, *) K_{S}(t) d m(t)
$$


The last conclusion follows readily. (Cf. [2].)

8. The Riesz representation theorem. A function $f(t, j)$ on $T \times J$ (not necessarily a quasi-function with respect to the measures $m_{j}, j \in J$ ) will be called measurable if $f(t, j)$ is measurable on $T$ for each $j$ in $J$; it is essentially bounded if there is a real number $r$ such that for each $j$ in $J,|f(t, j)| \leqq r$ except on an $m_{j}$-null set. The infimum of $r$ is the essential supremum of $|f(t, j)|$. If $f(t, j)$ and $f^{\prime}(t, j)$ are equivalent quasi-functions and one is essentially bounded, so is the other, and the essential suprema of their absolute values are equal. The set of all equivalence classes of essentially bounded quasifunctions will be denoted by $L_{\infty *}\left[T,\left\{m_{j}: j \in J\right\}\right]$, or by $L_{\infty *}$ when this will not cause confusion. If $\phi \in L_{\infty *}$ and $f(t, j) \in \phi$, we define $\|\phi\|_{\infty}$ to be the essential supremum of $|f(t, j)|$. As usual, if $f(t, j)$ is essentially bounded we shall (incorrectly, but conveniently) say that $f$ belongs to $L_{\infty *}$ and has norm $\|f(t, j)\|=$ ess. sup. $|f(t, j)|$.

If $b(t, *)$ is an essentially bounded quasi-function with respect to the measures $m_{R}(R \in R)$ or the measures $m_{L}(L \in \mathcal{L})$, it is easy to see that for every $m$-summable function $f(t)$ the integral

$$
l_{b}(f)=\int f(t) b(t, *) d m(t)
$$

exists and is finite. This defines a continuous linear functional $l_{b}$ over $L_{1}[T, m]$, and $\left\|l_{b}\right\|=\|b(t, *)\|$. We now prove the converse of this, which is a sort of extension of the Riesz representation theorem. (For a different kind of extension see [3].)

(8.1) TheOREM. Let $l$ be a continuous linear real-valued functional over $L_{1}[T, m]$. Then there exists an essentially bounded measurable quasi-function $b(t, L)$ with respect to $\left\{m_{L}: L \in \mathcal{L}\right\}$ such that $\|b(t, *)\|_{\infty}=\|l\|$ and for every function $f$ in $L_{1}[T, m]$

$$
l(f)=\int f(t) b(t, *) d m(t)
$$

The quasi-function $b(t, *)$ is unique up to equivalence.

By a well-known device we can represent $l$ as the difference of two nonnegative functionals, so without loss of generality we may assume $l$ nonnegative. For each $S$ in $a$ we define $n S$ to be the supremum of $l\left(K_{R \cap S}\right)$ for all $R$ in $R$; this is a countably additive measure on $Q$. By Theorem 7.1 there is a quasi-function $b(t, L)$ with respect to $\left\{m_{L}: L \in \mathscr{L}\right\}$ such that for each $S$ in $a$

$$
n S=\int b(t, *) K_{S}(t) d m(t)
$$


It is easy to see that the essential supremum of $|b(t, L)|$ is $\|l\|$. If $f$ is a "simple function," meaning that $f$ is a finite linear combination of characteristic functions of sets of finite measure, this implies

$$
l(f)=\int b(t, *) f(t) d m(t)
$$

Both members of this equation define continuous linear functions on $L_{1}[T, m]$, and the simple functions are dense in that space, so the equation holds for all $f$ in $L_{1}[T, m]$. 'The uniqueness of $b$, up to equivalence, is easy to establish.

9. Decomposition of a Hilbert space by means of a cyclic set. Let $T$ be a set and $a$ a $\sigma$-algebra of subsets of $T$. Let $H$ be a complex Hilbert space (not necessarily separable) and $\pi$ a resolution of the identity assigning to each set $S$ in $a$ a projector $\pi(S)$ in $H$ such that $\pi(T)$ is the identity $1, \pi(\varnothing)$ is the projector 0 on the origin, $\pi\left(S_{1}\right) \pi\left(S_{2}\right)=\pi\left(S_{1} \cap S_{2}\right)$ and $\pi$ is countably additive. For each $x$ in $H$ let $H(x)$ be the closure of the set of all linear combinations of projectors $\pi(S) x(S \in Q)$. If $y$ is orthogonal to $H(x)$, for all $S$ and $S^{\prime}$ in $a$ we have $\left(\pi(S) y, \pi\left(S^{\prime}\right) x\right)=\left(y, \pi\left(S \cap S^{\prime}\right) x\right)=0$, so $H(y)$ is orthogonal to $H(x)$. From the Hausdorff maximality principle we deduce that there is a set $\left\{x_{j}: j \in J\right\}$ of unit vectors in $H$ such that $H\left(x_{j}\right)$ and $H\left(x_{k}\right)$ are orthogonal whenever $j$ and $k$ are distinct members of $J$, and such also that the closed linear span of the $H\left(x_{j}\right)(j \in J)$ is $H$.

For all $j$ in $J$ and $S$ in $Q$ we define

$$
\mathrm{m}_{j} S=\pi(S) x_{j}, \quad m_{j} S=\left\|\pi(S) x_{j}\right\|^{2} .
$$

If $z$ is a simple function, with values $z_{1}, \cdots, z_{n}$ on disjoint measurable sets $S_{1}, \cdots, S_{n}$, we define

$$
\begin{aligned}
& \int z(t) d \mathrm{~m}_{j}(t)=\sum_{i=1}^{n} z_{i} \mathrm{~m}_{j} S_{i} \\
& \int z(t) d m_{j}(t)=\sum_{i=1}^{n} z_{i} m_{j} S_{i}
\end{aligned}
$$

Then

$$
\begin{aligned}
\left\|\int z(t) d \mathrm{~m}_{j}(t)\right\|^{2} & =\sum_{i}\left\|z_{i} \mathrm{~m}_{j} S_{i}\right\|^{2} \\
& =\sum_{i}\left|z_{i}\right|^{2} m_{j} S_{i} \\
& =\int|z|^{2} d m_{j}(t) .
\end{aligned}
$$

This isometry can be extended to all of (complex) $L_{2}\left[T, m_{j}\right]$; to each $z(t)$ in 
$I_{2}\left[T, m_{j}\right]$ there corresponds a unique vector-integral $\int z(t) d \mathrm{~m}_{j}(t)$, and its length is the norm of $z(t)$ in $L_{2}\left[T, m_{j}\right]$.

(9.1) Lemma. Let $H_{0}$ be the direct sum of the spaces $L_{2}\left[T, m_{j}\right](j \in J)$. For each function $z(t, j)$ in $H_{0}$ define

$$
\psi(z)=\sum_{j \in J} \int z(t, j) d \mathrm{~m}_{j}(t) .
$$

Then $\psi$ is an isometric linear mapping of $H_{0}$ onto $H$.

The linearity of $\psi$ is obvious. If $z(t, j) \in H_{0}$, for each $j$ in $J, z(t, j)$ is in $L_{2}\left[T, m_{j}\right]$ and therefore has an integral $y_{j}$ with respect to $\mathrm{m}_{j}$, and

$$
\left\|y_{j}\right\|^{2}=\int|z(t, j)|^{2} d m_{j}(t) .
$$

The $y_{j}$ are mutually orthogonal, and the sum of the right members is $\|z(t, j)\|^{2}$, so $\sum_{j} y_{j}$ exists and has length $\|z(t, j)\|$. Thus the mapping $\psi$ is defined on $H_{0}$ and is an isometry. The range of $\psi$ includes all vectors $\pi(S) x_{j}(S \in Q, j \in J)$, and the linear combinations of these are dense in $H$, so the range of $\psi$ is $H$.

For each bounded linear operator $B_{0}$ on $H_{0}$ we define

$$
\psi_{0}\left(B_{0}\right)=\psi B_{0} \psi^{-1} \text {. }
$$

Then $\psi_{0}$ is an isometric isomorphism of the algebra of all bounded linear operators on $H_{0}$ onto the algebra of all bounded linear operators on $H$, and is weakly continuous.

10. Multiplication operators. Each essentially bounded measurable function $f(t, j)$ on $T \times J$ (not necessarily a quasi-function on $T$ ) defines a bounded linear operator $f^{x}$ on $H_{0}$ by the relation

$$
f^{\times}(z)=f(t, j) z(t, j) \quad\left(z(t, j) \in H_{0}\right) .
$$

When $f(t, j)$ is a quasi-function on $T$ with respect to the measures $m_{j}(j \in J)$ the operator $f^{x}$ will be called a multiplication operator; the set of all multiplication operators will be denoted by $\mathfrak{T}$. It is obvious that $\mathfrak{T}$ is a commutative *-algebra over the complex field.

The algebra $\mathfrak{T}$ is mapped isometrically and isomorphically onto an algebra $\psi_{0}(\mathfrak{T})$ of operators in $H$. One special case is worth noting. Let $S$ be measurable, and let $q$ be the characteristic function of $S \times J$. Then $\psi_{0}\left(q^{\times}\right)$ $=\pi(S)$. For if $j \in J$ and $z$ is the characteristic function of a set $E \times\{j\}$ with $E$ in $a$, we have

so

$$
\psi(z)=\int z(t, j) d \mathrm{~m}_{j}(t)=\mathrm{m}_{j} E=\pi(E) x_{j}
$$

$$
\pi(S) \psi(z)=\pi(E \cap S) x_{j}=\mathrm{m}_{j}(E \cap S)=\psi(q(t, j) z(t, j))=\psi\left(q^{\times}(z)\right)
$$


This extends to linear combinations of such $z$, and by continuity to the closure of the set of linear combinations of such $z$, which is $H_{0}$. If $y \in H$ then there exists a $z$ in $H_{0}$ such that $y=\psi(z)$, so

$$
\pi(S) y=\psi\left(q^{\times}\left(\psi^{-1}(y)\right)\right)=\psi_{0}\left(q^{\times}\right) y .
$$

11. The multiplicative representation. We can now state our principal theorem on algebras of operators.

(11.1) THEOREM. With the notation of the two preceding sections,

(i) every bounded linear operator $C$ that commutes with every projector $\pi(S)(S \in Q)$ also commutes with all operators in $\psi_{0}(\Re)$;

(ii) the algebra $\psi_{0}(\mathfrak{T T})$ is weakly closed, and is the smallest weakly closed algebra of linear operators on $H$ that contains all the projectors $\pi(S)(S \in Q)$.

For any set $\mathfrak{N}$ of bounded linear operators on $H$ (or on $H_{0}$ ) let $\mathfrak{T}^{\prime}$ denote the set of all bounded linear operators on $H$ (or on $H_{0}$, respectively) that commute with every operator in $\Re$. Let $\Pi=\{\pi(S): S \in Q\}$. Since $\Pi \subset \psi_{0}(\mathfrak{T})$, it is clear that $\Pi^{\prime} \supset\left[\psi_{0}(\mathfrak{T})\right]^{\prime}$. Conclusion (i) is the assertion $\Pi^{\prime} \subset\left[\psi_{0}(\mathfrak{N})\right]^{\prime}$, and implies the equality of the two sets.

We shall use certain kinds of characteristic functions often enough to warrant abbreviation. If $j \in J$ and $S \subset T$, we shall use the symbols $K_{S, j}, K_{j}, K_{S}$ to denote the characteristic functions of the respective sets $S \times\{j\}, T \times\{j\}$, $S \times J$.

By $\S 10$, conclusion (i) is equivalent to $\left\{K_{S}^{\times}: S \in a\right\}^{\prime} \subset \mathfrak{M}^{\prime}$. Let then $C_{0}$ be an operator on $H_{0}$ that commutes with $K_{S}^{\times}$for all $S$ in $Q$; we must prove that $C_{0}$ commutes with all $q^{\times}$in $\Re$. So consider any $q(t, j)$ in $L_{\infty}{ }^{*}$. Let $z(t, j)$ belong to $H_{0}$, and let $y=C_{0} z$. Then $\int|z(t, j)|^{2} d m_{j}$ and $\int \mid y(t, j){ }^{2} d m_{j}$ vanish except for a countable subset $J_{c}$ of $J$. There is a bounded measurable function $q_{0}(t)$ such that for every $j$ in $J_{c}, q_{0}(t)=q(t, j)$ except on an $m_{j}$-null set. This $q_{0}(t)$ is the uniform limit of simple functions $q_{n}(t)(n=1,2, \cdots)$. Each $q_{n}$ is a finite linear combination

$$
q_{n}=\sum_{k=1}^{m_{n}} c_{n, k} K_{S_{n, k}} \quad\left(S_{n, k} \in a\right),
$$

so $q_{n}^{\times}$is a sum of operators each of which commutes with $C_{0}$. Hence

$$
C_{0}\left[q_{n}(t) z(t, j)\right]=q_{n}(t) C_{0}[z(t, j)]=q_{n}(t) y(t, j),
$$

so that

$$
C_{0}\left[q_{0}(t) z(t, j)\right]=q_{0}(t) y(t, j) .
$$

If $j \in J_{c}$, the equation

$$
q_{0}(t) z(t, j)=q(t, j) z(t, j)
$$

holds except on an $m_{j}$-null set, by definition of $q_{0}$; if $j \in J-J_{c}$, it holds except 
on the $m_{j}$-null set on which $z(t, j) \neq 0$. So the two members of the equation represent the same point of $H_{0}$. Similarly, $q_{0}(t) y(t, j)$ and $q(t, j) y(t, j)$ represent the same point of $H_{0}$, and the last equation of the preceding paragraph implies

$$
C_{0} \Upsilon^{\times}=q^{\times} C_{0} z .
$$

This holds for all $z$ in $H_{0}$, and (i) is established.

If $\Re$ is any set of bounded linear operators on $H$ such that for all $A$ in $\mathscr{N}$, $A^{*}$ is also in $\Re$, the smallest weakly closed algebra containing $\Re$ is $\Re^{\prime \prime}$ $\left[5\right.$, p. 44]. Since (i) holds, $\Pi^{\prime \prime}=\left[\psi_{0}(\mathscr{T})\right]^{\prime \prime}$, and (ii) will be established when we prove $\left[\psi_{0}(\mathfrak{T})\right]^{\prime \prime}=\psi_{0}(\mathfrak{T})$, or (more conveniently) $\mathfrak{T}^{\prime \prime}=\mathfrak{T}$. Let $A$ be any operator in $\mathfrak{T I}^{\prime \prime}$; we shall prove $A \in \mathfrak{T}$.

For $j \in J$, the characteristic function $K_{j}$ of $T \times\{j\}$ is in $H_{0}$, so $A K_{j}$ is an element $r_{j}(t, j)$ of $H_{0}$. Since $K_{j}^{\times}$is in $\mathfrak{T}^{\prime}$,

$$
\stackrel{\times}{K_{j} r_{j}}=A K_{j}^{\times}\left(K_{j}\right)=A K_{j}=r_{j} .
$$

We may therefore suppose that the chosen representation $r_{j}$ of $A K_{j}$ already has the property of vanishing except on $T \times\{j\}$, and we define

$$
r(t, j)=\sum_{j \in J} r_{j}(t, j)
$$

at each point in $T \times J$ at most one term in the sum is different from 0 .

If $j \in J$ and $S \in Q$ we have $K_{S, j}=K_{S, j} K_{j}$, so

$$
A\left(K_{S, j}\right)=A K_{S, j}^{\times}\left(K_{j}\right)=K_{S, j}^{\times} A\left(K_{j}\right)=K_{S, j}(t, j) r_{j}(t, j)=K_{S, j}(t, j) r(t, j) .
$$

Hence if $z(t, j)$ is a function assuming finitely many values, each on a set of the form $S \times\{j\}$ with $j \in J$ and $S \in a$, we obtain

$$
A(z)=z(t, j) r(t, j)=r^{\times}(z) .
$$

By the usual device we deduce that the essential supremum of $|r(t, j)|$ is $\|A\|$. Furthermore, the simple functions of the type just described are dense in $H_{0}$, and $A$ and $r^{\times}$are continuous, so the last equation implies $A=r^{\times}$. It remains to prove that $r$ is a quasi-function on $T$ with respect to the $m_{j}(j \in J)$, so that $r^{\times}$will belong to $\mathfrak{T}$.

Let $J_{c}$ be a countable subset of $J$; it can be mapped one-to-one on a set of positive integers by a function $(n(j): j \in J)$. For each $S$ in a define

$$
m S=\sum_{j \in J_{c}} 2^{-n(j)} m_{j} S .
$$

This is a countably additive non-negative measure on $a$, with $m(T) \leqq 1$; and for each $j$ in $J$ and each $S$ in $a, m_{j} S \leqq 2^{n(j)} m S$. By the Radon-Nikodym theorem, for each $j$ in $J$ there is a measurable function $f_{j}(t)$ on $T$, with $0 \leqq f_{j}$ $\leqq 2^{n(j)}$, such that for all $S$ in $a$ 


$$
\int_{S} f_{j}(t) d m(t)=m_{j} S
$$

With the standard definition of the signum function (so that $\operatorname{sgn} f_{j}(t)$ is 1 if $f_{j}(t)>0$ and is 0 if $\left.f_{j}(t)=0\right)$, for all $t$ in $T$ we define

$$
r(t)=\sup \left\{r_{j}(t, j) \operatorname{sgn} f_{j}(t): j \in J_{c}\right\} .
$$

This is measurable and essentially bounded. We shall now prove

$\left(^{*}\right)$ for each $j$ in $J_{c}, r(t)=r(t, j)$ except on an $m_{j}$-null set.

Suppose this false; for some $k$ in $J_{c}, r(t) \neq r(t, k)$ on a set $S_{1}$ with $m_{k} S_{1}>0$. Since $m_{k} S_{1}$ is the limit as $n$ increases of $m_{k}\left\{t: t \in S_{1}, f_{k}(t) \geqq 1 / n\right\}$, there is a subset $S_{2}$ of $S_{1}$ with $m_{k} S_{2}>0$ on which $f_{k}$ has a positive lower bound. By definition of $r$, this implies $r(t) \geqq r_{k}(t, k)$ on $S_{2}$, and they are unequal on $S_{1}$, so for each $t$ in $S_{2}$ there is a $j$ in $J_{c}$ for which $r_{j}(t, j)>r_{k}(t, k)$ and $f_{j}(t)>0$. Since $J_{c}$ is countable and $m S_{2}>0$, there is an $h$ in $J_{c}$ for which the set $S_{\mathbf{z}}$ $=\left\{t: t \in S_{2}, f_{h}(t)>0, r_{h}(t, h)>r_{k}(t, k)\right\}$ has positive $m$-measure. Since $f_{k}>0$ on $S_{3}, m_{k} S_{3}>0$. As in defining $S_{2}$, we find that there is a subset $S_{4}$ of $S_{3}$ with $m_{h} S_{4}>0$ on which $f_{h}(t)$ has a positive lower bound. Summarizing, $m_{h} S_{4}>0$ and $m_{k} S_{4}>0$; on $S_{4}$, both $f_{k}$ and $f_{k}$ have positive lower bounds and finite upper bounds; and on $S_{4}, r_{h}(t, h)>r_{k}(t, k)$.

Define $C$ on $H_{0}$ by setting $C z(t, j)=y(t, j)$, where

$$
\begin{aligned}
& y(t, j)=z(t, j) \text { unless } t \in S_{4} \text { and } j \in\{h, k\}, \\
& y(t, h)=z(t, k) \text { and } y(t, k)=z(t, h)\left(t \in S_{4}\right) .
\end{aligned}
$$

This is a bounded linear operator which is easily seen to commute with $K_{S}^{\times}$ for every $S$ in $a$, so by conclusion (i) $C$ is in $\mathfrak{N}^{\prime}$. Now let $z$ be the characteristic function of $S_{4} \times\{h\}$. Then at each point $(t, k)$ of $S_{4} \times\{k\}$ we have

$$
\begin{aligned}
C r^{\times}(z)(t, k) & =r_{h}(t, h), \\
r^{\times} C z(t, k) & =r_{k}(t, k),
\end{aligned}
$$

and the right members differ on all of $S_{4}$. So $r^{\times}$does not commute with the element $C$ of $9 \mathbb{T}^{\prime}$, and is therefore not in $\mathfrak{T}^{\prime \prime}$. But $r^{\times}=A$, and by hypothesis $A$ is in $\mathfrak{T}^{\prime \prime}$. This contradiction establishes $\left({ }^{*}\right)$, so $r(t, j)$ is a quasi-function and $A$ is in $\mathfrak{M}$. Thus $\mathfrak{N}^{\prime \prime} \subset \mathfrak{T}$, and the proof is complete.

12. The algebra of quasi-functions of operators. Consider now a weakly closed commutative *algebra $B$ of bounded operators on a Hilbert space $H$ (a von Neumann algebra, in Dixmier's terminology). Let $\left(B_{\omega}: \omega \in \Omega\right.$ ) be a set of hermitian operators in $B$ such that the smallest weakly closed algebra containing the set is $B$ itself. Let $T_{\omega}$ be the interval $\left[-\left\|B_{\omega}\right\|,\left\|B_{\omega}\right\|\right]$, and let $T$ be the cartesian product $\chi_{\omega} T_{\omega}$. By a proof in $[4, \mathrm{pp} .125$ et seq.] we know that there exists a $\sigma$-field $a$ of subsets of $T$ including all Borel subsets of $T$, and on $a$ a projector-valued measure $(\pi(S): S \in Q)$ which is a resolution of the identity, such that for each $\omega$ in $\Omega$ the integral of the coordinate-function 
$t_{\omega}$ with respect to $\pi$ is $B_{\omega}$. Whenever $q(t)$ is bounded and measurable the integral $\int q(t) d \pi(t)$ exists and is a bounded operator on $H$, which we denote by $q\left(B_{w}\right)$ to indicate its dependence on all the $B_{\omega}$; these operators obey the usual rules of an operational calculus. As we shall shortly prove, the set of all such $q\left(B_{w}\right)$ is contained in $B$. However, it may be a proper subset of $B$ and may fail to be weakly closed, as we now show.

Let $U=[-1,1], U^{2}=U \times U$. Let $m_{1}$ be one-dimensional Lebesgue measure, and let $a$ be the class of all subsets of $U^{2}$ that meet each ordinate and each abscissa in an $m_{1}$-measurable set. For each $S$ in $a$ we define

$$
m S=\sum_{u \in U} m_{1}(S \cap[\{u\} \times U])+\sum_{u \in U} m_{1}(S \cap[U \times\{u\}]) .
$$

This system satisfies (1.1). Let $H$ be $L_{2}\left[U^{2}, m\right]$. The operator $B$ that transforms $z$ into $\left(u_{1} z\left(u_{1}, u_{2}\right):\left(u_{1}, u_{2}\right) \in U^{2}\right)$ is hermitian and has bound 1 , so its spectrum is contained in $T=[-1,1]$. Let $\pi$ be the resolution of the identity for $B$, and let $B$ be the smallest weakly closed algebra of operators on $H$ that contains $B$. For each $\tau$ in $T$ define

$$
f_{n, \tau}(t)=\left[1-(t-\tau)^{2} / 5\right]^{n}
$$

As $n$ increases this converges monotonically to the characteristic function of $\{\tau\}$. Hence $\pi(\{\tau\})$ is the weak limit of the $f_{n, \tau}(B)$ and is therefore in $B$. So too is $\pi(F)$ for every finite subset $F$ of $T$, and by $[4$, p. 108] so is

$$
C=\sup \{\pi(F): F \text { finite, } F \subset T\} \text {. }
$$

It is easy to show that $\pi(\{\tau\})$ is the operation of multiplying by the characteristic function of $\{\tau\} \times U$, so for each $z$ in $H$ the transform $w=C z$ is defined by

$$
\begin{aligned}
w\left(u_{1}, u_{2}\right) & =z\left(u_{1}, u_{2}\right) \text { for all } u_{1} \text { such that } \int_{U}\left|z\left(u_{1}, v\right)\right|^{2} d v>0 \\
& =0 \text { for all other } u_{1} .
\end{aligned}
$$

This operator cannot be represented in the form $\int f(t) d \pi(t)$ with any function $f$. For suppose $C$ thus represented. For each $\tau$ in $T$, let $z$ be the characteristic function of $\{\tau\} \times U$. This is in $H$, and $\pi(\{\tau\}) z=z$. Hence $\pi\left(T^{\prime}\right) z=0$ whenever $T^{\prime} \subset T-\{\tau\}$, and

$$
z=C z=\left[\int_{T} f(t) d \pi(t)\right] z=f(\tau) z,
$$

whence $f(\tau)=1$. This holds for all $\tau$ in $T$, so $C$ is the identity operator. But if $z$ is the characteristic function of $U \times\{0\}, C z=0 \neq z$. So $C$ is not represented as an integral, and the class of functions $q(B)$ is not weakly closed.

We correct this inadequacy by enlarging the class of integrands. Let the cyclic set $\left\{x_{j}: j \in J\right\}$, the subspaces $H\left(x_{j}\right)$ and the measures $m_{j}$ be as in $\S 9$ 
and let $P_{j}$ be the projector on $H\left(x_{j}\right)$. If $q(t, j)$ is measurable and essentially bounded (cf. §8) the expression

$$
q\left(B_{w}\right)=\sum_{j \in J} \int q(t, j) d \pi(t) P_{j}
$$

defines a bounded linear operator on $H$; for if $y \in H, P_{j} y=0$ except for countably many $j$, and the sum

$$
\sum_{j \in J} \int q(t, j) d \pi(t) P_{j} y
$$

converges in norm to a limit whose norm is at most ess. sup. $|q(t, j)| \cdot\|y\|$, and the linearity is evident. However, we now have too many operators. Easy examples, even with two-dimensional $H$, show that $q\left(B_{w}\right)$ may fail to belong to $B$. This excess is remedied by restricting the $q(t, j)$ to be essentially bounded quasi-functions on $T$, that is to belong to $L_{\infty *}=L_{\infty *}\left[T,\left\{m_{j}: j \in J\right\}\right]$. It is trivially easy to prove all but the last conclusion of the following theorem.

(12.1) THEOREM. For each quasi-function $q(t, j)$ in $L_{\infty *}$, the operator $q\left(B_{w}\right)$ just defined is linear and has bound ess. sup. $|q(t, j)|$; and $\bar{q}\left(B_{w}\right)$ $=\left[q\left(B_{w}\right)\right]^{*}$. If $q_{1}(w, j)$ and $q_{2}(t, j)$ are in $L_{\infty *}$ and $c_{1}$ and $c_{2}$ are complex numbers, and $q_{3}(t, j)=c_{1} q_{1}(t, j)+c_{2} q_{2}(t, j)$ and $q_{4}(t, j)=q_{1}(t, j) q_{2}(t, j)$, then

$$
q_{3}\left(B_{w}\right)=c_{1} q_{1}\left(B_{w}\right)+c_{2} q_{2}\left(B_{w}\right) \text { and } q_{4}\left(B_{w}\right)=q_{1}\left(B_{w}\right) q_{2}\left(B_{w}\right) \text {. }
$$

If $q(t, j)$ is equivalent to $q_{0}(t)$, then $q\left(B_{w}\right)$ is the same as $q_{0}\left(B_{w}\right)$ as ordinarily defined. The set of all operators $q\left(B_{w}\right)\left(q(t, j) \in L_{\infty *}\right)$ is the algebra $B$.

Let $q(t, j)$ belong to $L_{\infty *}$. For each $C$ in $B^{\prime}$, to each $y$ in $H$ there corresponds a countable subset $J_{c}$ of $J$ such that $P_{j} y=P_{j} C y=0$ for all $j$ in $J-J_{c}$. There is a measurable $f(t)$ such that $f(t)=q(t, j)$ except on an $m_{j}$-null set for all $j$ in $J_{c}$, and by classical theory $f\left(B_{w}\right)$ commutes with $C$. Thus $C q\left(B_{w}\right) y=C f\left(B_{w}\right) y=f\left(B_{w}\right) C y=q\left(B_{w}\right) C y$. Since $y$ is arbitrary in $H, q\left(B_{w}\right)$ commutes with the arbitrary member $C$ of $B^{\prime}$, and is therefore in $B^{\prime \prime}$. It remains to prove that every member of $B$ can be written in the form $q\left(B_{w}\right)$ for some $q$ in $L_{\infty *}$.

Let $K_{S}$ be the characteristic function of a measurable subset $S$ of $T$. By $\S 10, \psi_{0}\left(K_{S}^{\times}\right)=\pi(S)$, so when $f=K_{S}$ the equation

$$
\int f(t) d \pi(t)=\psi_{0}\left(f^{\times}\right)
$$

is valid. By linearity it holds for all simple functions. If $f(t)$ is bounded and measurable it is the uniform limit of a sequence $s_{1}, s_{2}, \ldots$ of simple functions. Then $s_{n}^{\times}$tends to $f^{\times}$in the uniform topology of operators, so $\psi_{0}\left(s_{S}^{\times}\right)$tends in 
the same manner to $\psi_{0}\left(f^{\times}\right)$. Also, $\int s_{n}(t) d \pi(t)$ tends in the same manner to $\int f(t) d \pi(t)$, so $\left({ }^{* *}\right)$ holds for all bounded measurable functions $f(t)$.

Let $q(t, j)$ belong to $L_{\infty *}$. For each $z(t, j)$ in $H_{0}$, let $z_{j}$ be the function on $T \times J$ defined by $z_{j}(t, k)=z(t, j)$ if $k=j, z_{j}(t, k)=0$ otherwise. By Lemma 9.1, the vector $y=\psi(z)$ of $H$ is expressed as the sum of integrals, each of which is a vector in the correspondingly labeled subspace $H\left(x_{j}\right)$. Hence

$$
P_{j} y=\int z(t, j) d \mathbf{m}_{j}(t)=\psi\left(z_{j}\right) \quad(j \in J) .
$$

From this and $(* *)$, with the definition of $\psi_{0}$,

$$
\begin{aligned}
{\left[\int q(t, j) d \pi\right] P_{j} y } & =\psi_{0}\left(q(t, j)^{\times}\right) \psi\left(z_{j}\right) \\
& =\psi\left(q(t, j)^{\times} z_{j}\right) \quad(j \in J) .
\end{aligned}
$$

Summing over all $j$ in $J$ yields

$$
q\left(B_{\mathbf{w}}\right) y=\psi\left(q^{\times}\right)
$$

or

$$
\psi^{-1}\left(q\left(B_{w}\right) \psi(z)\right)=q^{\times} z
$$

that is, $q\left(B_{w}\right)=\psi_{0}\left(q^{\times}\right)$. This last is an arbitrary member of the algebra $\psi_{0}(\mathfrak{T})$. By Theorem 11.1 this algebra is weakly closed, and it clearly contains all the $B_{w}$, so it contains all of $B$. Thus each operator in $B$ can be represented in the form $q\left(B_{w}\right)$ for some $q$ in $L_{\infty *}$, and the proof is complete.

As a corollary, if the spectrum has countable multiplicity (that is, if $J$ is countable) the set of operators $f\left(B_{w}\right)$ with $f$ essentially bounded, measurable and complex-valued on $T$ is identical with the algebra $B$.

\section{REFERENCES}

1. Paul Halmos, Measure theory, Van Nostrand, New York, 1950.

2. I. E. Segal, Equivalence of measure spaces, Amer. J. Math. 73 (1951), 275-313.

3. J. Schwartz, A note on the spaces $L_{p}^{*}$, Proc. Amer. Math. Soc. 2 (1951), 270-275.

4. E. J. McShane, Order-preserving maps and integration processes, Princeton Univ. Press, Princeton, N. J., 1954. 1957.

5. J. Dixmier, Les algèbres d'opérateurs dans l'espace hilbertien, Gauthier-Villars, Paris,

Added in proof:

6. A. C. Zaanen, The Radon-Nikodym theorem, Nederl. Akad. Wetensch. Proc. Ser. A 64 (1961), 156-187.

UNIVERSITY OF ViRGINIA,

Charlottesville, Virginia 